\title{
ОДНОЕЛЕКТРОННІ ОПТИЧНІ \\ ВЛАСТИВОСТІ МЕТАЛЕВИХ НАНООБОЛОНОК 3 НЕКОНЦЕНТРИЧНИМ ЯДРОМ. ВРАХУВАННЯ КВАНТУВАННЯ ЕЛЕКТРОННОГО СПЕКТРА
}

Роботу присвячено дослідженню одноелектронних оптичних властивостей (внесок одноелектронної компоненти є суттєвим у областях частот, далеких від плазмонного резонансу) сферичної наночастинки, що складається з діелектричного ядра та тонкої металевої оболонки, зі слабким зміщенням центра ядра відносно геометричного центра всієї наночастинки. Запропоновано модель, що дозволяє записати хвильові функції та спектр хвильових чисел для електрона у оболонці композитної наночастинки такого типу. За допомогою цієї моделі отримано матричні елементи оптичних переходів та одноелектронну оптичну провідність наночастинки як без урахування (напівкласична провідність), так і з урахуванням квантування хвильових чисел електрона у оболонці. Показано, що врахування вищеназваних ефектів квантування приводить до появи осцилюючої залежності оптичної провідності наночастинки від частоти світла. Показано, що вплив зміщення центрів та квантування спектра на оптичну провідність наночастинки у першому наближенні можна враховувати незалежно.

\section{1. Вступ}

Розвиток нанотехнологій у останні десятиріччя дозволив використання у техніці наночастинок різних конфігурацій i, відповідно, з різними властивостями. Зокрема у техніці широко використовуються металеві наночастинки; їх застосування грунтуються, передусім, на унікальних оптичних властивостях. Так, ці частинки ефективно поглинають світло на заданій довжині хвилі. Композитні наночастинки такого типу, отримані у останні роки - так звані нанооболонки [1-4] - сприяють розвитку цих технологій, розширюючи робочий діапазон довжин хвиль порівняно 3 традиційними суцільними металевими наночастинка- ми. Нанооболонки - це композитні наночастинки, що складаються з діелектричного ядра і тонкої металевої оболонки. Зауважимо також, що оптичні властивості нанооболонок можна регулювати більш гнучко, ніж у традиційних системах. Така гнучкість пов'язана 3 тим, що внутрішній та зовнішній радіуси металевої оболонки (оптичний відгук якої є визначальним для всієї частки) можуть змінюватись незалежно. Це робить нанооболонки особливо перспективними для технічних застосувань.

Після отримання нанооболонок у останні роки їх активно досліджували як теоретично [5-11] (з використанням класичного і квантового підходу), так і експериментально $[3,5,12,13]$. Проте їх оптичні властивості вивчали переважно у околі плазмонного резонансу. Але у областях частот, далеких від плазмонного резонансу, внесок індивідуальних переходів у поглинання світла стає домінуючим. Роботи, що вивчають внесок індивідуальних переходів у поглинання світла, як правило, розглядають один з двох протилежних граничних випадків. Перший випадок відповідає ситуації, коли відстані між квантовими електронними рівнями дуже малі порівняно з енергією кванта світла і сума по дискретних рівнях замінюється інтегралом. Другий граничний випадок відповідає ситуації, коли відстань між енергетичними рівнями одного порядку з енергією кванта світла, так що враховуються тільки два-три рівні. Проте електронних рівнів у типовій оболонці набагато більше. 3 іншого боку, як показано у даній роботі, квантовими ефектами, що пов'язані з квантуванням енергії електрона у оболонці, нехтувати не можна. Причина цього полягає в то- 
му, що енергетичні рівні для тонкої оболонки стають квазіодновимірними, так що відстань між ними зростає.

Як ми покажемо у роботі, врахування таких розмірних квантових ефектів приводить до появи осцилюючої залежності оптичних властивостей нанооболонки від товщини металевого покриття. Осцилюючу залежність - як оптичних, так і електричних властивостей - металевого нанодроту від його товщини, подібну до отриманої у даній роботі, спостерігали експериментально у [14]. Подібний вигляд залежності для суцільного металевого нанодроту, товщина якого є відносно великою, означає, що подібні квантові ефекти для тонких оболонок є набагато сильнішими.

Зауважимо, що у малих металевих частинках (i, зокрема, у металевих нанооболонках) при дослідженні поглинання світла потрібно досліджувати обидві компоненти поглинання - як електричне (пов'язаним з електричним вектором електромагнітної хвилі), так і магнітне. Це випливає з того, що у таких частинках може домінувати як магнітне, так і електричне поглинання залежно від форми і розміру частинки та частоти електромагнітної хвилі [15]. Проте у попередніх роботах, присвячених дослідженню поглинання світла у таких оболонках, вивчали передусім магнітне поглинання (див., наприклад, [16-18]). Це робить дослідження електричного поглинання у металевих оболонках різного типу актуальним.

Відомо, що одна з унікальних властивостей наночастинок полягає у залежності оптичних властивостей частинки від їі форми та розміру (див., наприклад, [15]). Тому оптичні властивості досліджуються окремо для наночастинок різних форм як суцільних, так i композитних, що становлять ще ширший діапазон конфігурацій.

У даній роботі досліджено різновид композитних наночастинок, що потрапив у поле зору дослідників порівняно нещодавно - так звані нанояйця [19-21]. Нанояйцями називають звичайні (близькі до сферичної форми) нанооболонки з порушенням симетрії, тобто нанооболонки, центр ядра якої зміщено відносно центра всієї наночастинки. Крім зовнішнього розміру та відношення розмірів діелектричного ядра та оболонки, параметром, що визначає оптичні властивості нанояйця, є також і відносне зміщення центрів ядра та наночастинки. Отже, такі композитні наночастинки дають ще більш широкий діапазон застосувань, і їх оптичні властивості можуть бути змінені ще більш гнучко, ніж для симетричних композитних наночастинок. Метою даної роботи є теоретичне дослідження оптичних властивостей деформованого еліпсоїда зі слабким зміщенням центра діелектричного ядра відносно геометричного центра наночастинки, тобто зі слабким порушенням симетрії. Конкретно у роботі буде знайдено оптичну провідність (з урахуванням електричного поглинання) названих вище оболонок у одноелектронному наближенні для частот, далеких від плазмонного резонансу. При обчисленні оптичної провідності враховано дискретність електронних рівнів енергіі, що робить отримані результати застосовними навіть для дуже тонких оболонок.

\section{2. Постановка задачі. Робоча модель}

Розглянемо деформований еліпсоїд, що складається 3 діелектричного ядра та тонкої металевої оболонки. Нехай деформований еліпсоїд - як його зовнішня границя наночастинки, так і границя діелектричного ядра - має форму, близьку до сферичної. Позначимо внутрішній та зовнішні радіуси оболонки $a$ і $b$ відповідно. Ми розглядаємо випадок тонкої оболонки, так що виконується $\frac{b-a}{b} \ll 1$.

$\mathrm{У}$ даній роботі розглядаємо наночастинка, внутрішній та зовнішній центри якого (тобто геометричні центри діелектричного ядра та зовнішньої границі оболонки відповідно) зміщено один відносно іншого на малу величину $\Delta l$, так що виконується $\frac{\Delta l}{a} \ll 1$, $\frac{\Delta l}{b-a} \ll 1$, а, отже, i $\frac{\Delta l}{b} \ll 1$. Для врахування такого малого зміщення застосуємо спочатку відому модель симетричної сферичної нанооболонки [22] і далі знайдемо малу поправку до оптичної провідності оболонки, зумовлену відносним зміщенням центрів оболонки. Згідно з [22] розглядаємо сферичну металеву оболонку (діелектричне ядро не дає суттєвого внеску у провідність частинки), моделюючи її безмежно глибокою потенційною ямою для електронів (можливість застосування такої моделі випливає з співвідношення між енергією Фермі та роботою виходу для типових металів) і застосовуючи одноелектронну модель.

Нехай на описаний вище деформований еліпсоїд падає електромагнітна хвиля, довжина якої набагато більша за зовнішній розмір оболонки, так що поле хвилі у оболонці можна вважати однорідним; оберемо вісь $O z$ у напрямку поширення хвилі. Розглянемо випадок, коли цей напрямок збігається з напрямком зміщення центрів у оболонці. Тоді у відповідній сферичній системі координат $(r, \theta, \phi)$ з формулами пере- 
ходу до декартових:

$$
\left.\begin{array}{l}
x=r \cos \varphi \sin \theta \\
y=r \sin \varphi \sin \theta \\
z=r \cos \theta
\end{array}\right\}
$$

мале зміщення $\Delta l$ центрів можна описати, замінивши

$b \rightarrow b(1 \pm \alpha \cos \theta), \quad \alpha=\frac{\Delta l}{b} \ll 1$,

знак "+" відповідає випадку, коли центр зовнішньої сфери зміщено відносно внутрішнього у напрямку поширення хвилі, "-_ - у протилежному.

3 умови $\frac{\Delta l}{b-a} \ll 1$ випливає, що товщина оболонки змінюється повільно та на малу величину. Оскільки задача про знаходження хвильових функцій, спектра енергії та хвильових чисел електрона у тонкій оболонці зводиться до квазіодновимірної задачі (електрон у одновимірній потенційній ямі), то при малій та повільній зміні товщини можемо для кожного напрямку $(\theta, \phi)$ використати відповідний вираз для сферичної оболонки. Отже, при знаходженні поправки до оптичної провідності, пов'язаної з малим зміщенням центрів, ми можемо використовувати хвильові функції та спектр хвильових чисел сферичної нанооболонки у слабко деформованій (відносно сферичної $r=a, r=b)$ потенційній ямі, границі якої у сферичних координатах задаються рівняннями $r=a$, $r=b(1 \pm \alpha \cos \theta)$ з подальшою підстановкою цих хвильових функцій та спектра у матричний елемент оптичного переходу.

Отже, задача даної роботи полягає у знаходженні оптичної провідності описаної вище наночастинки при падінні на неї електромагнітної хвилі з великою (порівняно з розміром наночастинки) довжиною хвилі.

\section{3. Вираз для оптичної провідності через суму по електронних станах}

Для розрахунку оптичної провідності наночастинки, описаної у попередньому розділі, використаємо метод, який наведено у [22]. Запишемо спочатку вираз для компонент провідності нанооболонки через матричні елементи оптичного переходу електрона. Оптичну провідність у напрямку поширення хвилі (вісь $O z)$ запишемо так:

$$
\begin{aligned}
& \sigma=\frac{\pi e^{2} \omega}{V_{s}} \sum_{i, f}|\langle i|z| f\rangle|^{2} f\left(E_{i}\right)\left(1-f\left(E_{f}\right)\right) \times \\
& \times \delta\left(E_{f}-E_{i}-\hbar \omega\right),
\end{aligned}
$$

де $V_{s}$ - об'єм нанооболонки, $\omega$ - частота хвилі; індексом $i$ позначено початковий, $f$ - кінцевий стан електрона; $E_{i}$ та $E_{f}$ - енергія електрона у початковому та кінцевому станах відповідно; $f(E)$ - електронна функція розподілу по енергіях.

Для знаходження матричних елементів застосуємо методику, наведену в [22]. Ми знайдемо хвильові функції та спектр хвильових чисел для електрона у металевій оболонці, використовуючи їх, обчислимо матричні елементи оператора координати $z$ i, нарешті, просумувавши по початкових та кінцевих станах електрона, отримаємо власне провідність.

Розглянемо спочатку симетричну сферичну оболонку. Хвильові функції та спектр хвильових чисел для такої оболонки отримано після розділення у сферичних координатах (1) - змінних у рівнянні Шредінгера для електрона у безмежній потенційній ямі. Згідно з [22] після розв'язку рівняння Шредінгеpa, підстановки граничних умов та врахування тонкості оболонки $\left(\frac{b-a}{b} \ll 1\right)$ хвильову функцію $\psi$ та спектр хвильових чисел $k$ сферичної оболонки записуємо

$\psi(r, \theta, \varphi) \approx$

$\approx\left(\frac{2}{b-a}\right)^{1 / 2} \frac{1}{r} \sin \left(k r-(l+1) \frac{\pi}{2}+\alpha\right) Y_{l m}(\theta, \varphi)$,

$k=\frac{\pi n}{b-a}$,

де $Y_{l m}$ - поліноми Лежандра, $a, b$ - внутрішній та зовнішній радіуси потенційної ями, відповідно - відповідають, очевидно, внутрішньому та зовнішньому радіусам металевої оболонки.

Заміна (2) приведе до зміни матричного елемента оптичного переходу у напрямку поширення хвилі. Матричний елемент для сферичної нанооболонки запишемо

$\left.\left.\langle i|z| f\rangle=\int_{0}^{2 \pi} d \varphi \int_{0}^{\pi} \sin \theta d \theta \int_{a}^{b} r^{2} d r Y_{l m} \theta, \varphi\right) Y_{l^{\prime} m^{\prime}}^{*} \theta, \varphi\right) \times$

$\times \frac{2}{b-a} \frac{1}{r^{2}} \sin \left(k r-(l+1) \frac{\pi}{2}+\alpha\right) \times$

$\times \sin \left(k^{\prime} r-\left(l^{\prime}+1\right) \frac{\pi}{2}+\alpha^{\prime}\right) r \cos \theta$. 
Зауважимо, що в цей вираз входить різниця $b-a$ у степені -1. У інтеграл по радіальній змінній

$$
\begin{aligned}
& \int_{a}^{b} r \sin \left(k r-(l+1) \frac{\pi}{2}+\alpha\right) \times \\
& \times \sin \left(k^{\prime} r-\left(l^{\prime}+1\right) \frac{\pi}{2}+\alpha^{\prime}\right) d r= \\
& =\frac{1-(-1)^{n+n^{\prime}}}{2} \frac{2 k^{\prime} 2 k}{\left(\left(k^{\prime}\right)^{2}-k^{2}\right)^{2}},
\end{aligned}
$$

зважаючи на те, що з 3$) \frac{k k^{\prime}}{\left(\left(k^{\prime}\right)^{2}-k^{2}\right)^{2}} \sim(b-a)^{2}$, входить квадрат різниці $b-a$. Отже, остаточно можна записати, що після заміни (2) у матричному елементі (6) інтеграл по радіальних змінних зміниться пропорційно величині $(b-a)^{2}(b-a)^{-1}=b-a \rightarrow$ $(b-a)\left(1 \pm \frac{\alpha b}{b-a} \cos \theta\right)$, тобто для інтеграла по радіальних змінних, що входить у матричний елемент (6),

$$
\begin{aligned}
& \int_{0}^{2 \pi} d \varphi \int_{0}^{\pi} d \theta Y_{l m}^{*}(\theta, \varphi) \cos \theta Y_{l^{\prime} m^{\prime}}(\theta, \varphi) \sin \theta \rightarrow \int_{0}^{2 \pi} d \varphi \times \\
& \times \int_{0}^{\pi} d \theta Y_{l m}^{*}(\theta, \varphi) \cos \theta Y_{l^{\prime} m^{\prime}}(\theta, \varphi) \times
\end{aligned}
$$$$
\times \sin \theta\left(1 \pm \frac{\alpha b}{b-a} \cos \theta\right)
$$

Обчислимо цей інтеграл. Введемо для зручності позначення $\frac{\alpha b}{b-a}=\beta .3$ відомих формул для поліномів Лежандра

$$
\left\{\begin{array}{l}
Y_{l m}(\theta, \varphi) \equiv \sqrt{\frac{2 l+1}{4 \pi} \frac{(l-m) !}{(l+m) !}} P_{l}^{m}(\cos \theta) e^{i m \varphi} \\
x P_{l}^{m}(x)=\frac{l-m+1}{2 l+1} P_{l+1}^{m}+\frac{l+m}{2 l+1} P_{l-1}^{m}
\end{array}\right.
$$

можна записати таке рекурентне співвідношення:

$$
\begin{aligned}
& Y_{l m}(\theta, \varphi) \cos \theta=\sqrt{\frac{2 l+1}{4 \pi} \frac{(l-m) !}{(l+m) !}} P_{l}^{m}(\cos \theta) e^{i m \varphi} \cos \theta= \\
& =\sqrt{\frac{2 l+1}{4 \pi} \frac{(l-m) !}{(l+m) !}} e^{i m \varphi}\left(\frac{l-m+1}{2 l+1} P_{l+1}^{m}+\frac{l+m}{2 l+1} P_{l-1}^{m}\right)=
\end{aligned}
$$

$=\sqrt{\frac{(l+m+1)(l-m+1)}{(2 l+1)(2 l+3)}} Y_{l+1}^{m}+$

$+\sqrt{\frac{(l+m)(l-m)}{(2 l+1)(2 l-1)}} Y_{l-1}^{m}$.

3 ціеї властивості поліномів Лежандра та їх ортогональності знаходимо

$\int_{0}^{2 \pi} d \varphi \int_{0}^{\pi} d \theta Y_{l m}^{*}(\theta, \varphi) \cos \theta Y_{l^{\prime} m^{\prime}}(\theta, \varphi) \sin \theta(1 \pm \beta \cos \theta)=$ $=\int_{0}^{2 \pi} d \varphi \int_{0}^{\pi} d \theta\left(\gamma(l+1, m) Y_{l+1, m}^{*}+\gamma(l, m) Y_{l-1, m}^{*} \pm\right.$

$\pm \beta\left(\gamma(l+1, m) \gamma(l+2, m) Y_{l+2, m}^{*}+\left(\gamma^{2}(l+1, m)+\gamma^{2} \times\right.\right.$

$\left.\left.\times(l, m)) Y_{l, m}^{*}+\gamma(l, m) \gamma(l-1, m) Y_{l-2, m}^{*}\right)\right) Y_{l^{\prime} m^{\prime}}(\theta, \varphi) \sin \theta=$

$=\delta_{m, m^{\prime}}\left(\gamma\left(l^{\prime}, m^{\prime}\right) \delta_{l, l^{\prime}-1}+\gamma(l, m) \delta_{l, l^{\prime}+1} \pm\right.$

$\pm \beta\left(\gamma\left(l^{\prime}, m^{\prime}\right) \gamma\left(l^{\prime}-1, m^{\prime}\right) \delta_{l, l^{\prime}-2}+\right.$

$+\left(\gamma^{2}(l+1, m)+\gamma^{2}(l, m)\right) \delta_{l, l^{\prime}}+$

$\left.\left.+\gamma(l, m) \gamma(l-1, m) \delta_{l, l^{\prime}-2}\right)\right)$.

Тут введено функцію $\gamma$ двох цілих аргументів:

$\gamma(l, m) \equiv \sqrt{\frac{(l+m)(l-m)}{(2 l+1)(2 l-1)}}$.

Проінтегрувавши таким чином по кутових та радіальних змінних, підставимо отримане значення матричного елемента $<i|z| f>$ у вираз для провідності (3). Через ортогональність дельта-індексів отримаємо $\sigma=\frac{2 \pi e^{2} \hbar^{4}}{V_{s} m_{e}^{4} \omega^{3}} \frac{1}{(b-a)^{2}} \sum_{n, l, m, n^{\prime}, l^{\prime}, m^{\prime}}\left(1-(-1)^{n+n^{\prime}}\right) k^{2}\left(k^{\prime}\right)^{2} \times$ $\times f\left(E_{i}\right)\left(1-f\left(E_{f}\right)\right) \delta\left(E_{f}-E_{i}-\hbar \omega\right) \delta_{m, m^{\prime}}\left(\gamma^{2}\left(l^{\prime}, m^{\prime}\right) \delta_{l, l^{\prime}-1}+\right.$ $+\gamma^{2}(l, m) \delta_{l, l^{\prime}+1} \pm \beta^{2}\left(\gamma^{2}\left(l^{\prime}, m^{\prime}\right) \gamma^{2}\left(l^{\prime}-1, m^{\prime}\right) \delta_{l, l^{\prime}-2}+\right.$ 
$+\left(\gamma^{2}(l+1, m)+\gamma^{2}(l, m)\right)^{2} \delta_{l, l^{\prime}}+$

$\left.\left.+\gamma^{2}(l, m) \gamma^{2}(l-1, m) \delta_{l, l^{\prime}-2}\right)\right)$

Підсумуємо спочатку по квантових числах $l, l, m$ та $m^{\prime}$. Як відомо, $m$ змінюється в межах $-l \leq m \leq l, l$ - від 0 до $2 \mathrm{n}$. Наявність дельта-індексів $\delta_{m, m^{\prime}}$ i $\overline{\delta_{l, l^{\prime}+1}}$ змінюе картину підсумовування - фактично $l$ і $l$ ' змінюються від 0 до $2 \min \left(n, n^{\prime}\right) \pm 1, m-$ від $-\min \left(l, l^{\prime}\right)$ до $\min (l, l$ '). Якщо, як у нашому випадку, наявне поглинання, $n$ має менше значення, ніж $n^{\prime}$, тому $0 \leq l$, $l ' \leq 2 n$. Тут ми знехтували одиницею порівняно з $2 n$, оскільки підсумовування йде по вузькій області термічного розмиття поблизу енергії Фермі, в якій $n \gg 1$. Звідси також випливає, що доданки з малими $l, l, m$ та $m^{\prime}$ не можуть давати суттєвий внесок у результуючу суму, так що ми можемо вважати ці квантові числа набагато більшими за одиницю і, зокрема, $\gamma^{2}(l, m) \approx \frac{l^{2}-m^{2}}{4 l^{2}}$. Після врахування цих фактів підсумовування по $l, l, m$ та $m^{\prime}$ можна провести так:

$\sum_{l, m, l^{\prime}, m^{\prime}} \delta_{m, m^{\prime}}\left(\gamma^{2}\left(l^{\prime}, m^{\prime}\right) \delta_{l, l^{\prime}-1}+\gamma^{2}(l, m) \delta_{l, l^{\prime}+1} \pm\right.$

$\pm \beta^{2}\left(\gamma^{2}\left(l^{\prime}, m^{\prime}\right) \gamma^{2}\left(l^{\prime}-1, m^{\prime}\right) \delta_{l, l^{\prime}-2}+\left(\gamma^{2}(l+1, m)+\right.\right.$

$\left.\left.\left.+\gamma^{2}(l, m)\right)^{2} \delta_{l, l^{\prime}}+\gamma^{2}(l, m) \gamma^{2}(l-1, m) \delta_{l, l^{\prime}-2}\right)\right) \approx$

$\approx \sum_{l, m, l^{\prime}, m^{\prime}} \delta_{m, m^{\prime}} \delta_{l, l^{\prime}}\left(2 \gamma^{2}(l, m) \pm 6 \beta \gamma^{4}(l, m)\right)=$

$=\sum_{m=-\tilde{l}}^{\tilde{l}}\left(2 \gamma^{2}(\tilde{l}, m) \pm 6 \beta \gamma^{4}(l, m)\right) \approx$

$\approx \frac{2 \tilde{l}}{3} \pm 6 \beta \frac{\tilde{l}}{5}=\frac{2 \tilde{l}}{3}\left(1 \pm \frac{3}{5} \beta^{2}\right)$

де позначено $\tilde{l}=\min \left(l, l^{\prime}\right)$.

Бачимо, що врахування зміщення центрів у деформованому еліпсоїді приводить до появи сталого множника $1 \pm \frac{3}{5} \beta^{2}$ у виразі для провідності вже на цьому етапі. Підсумовуючи далі по $\tilde{l}$

$\sum_{\tilde{l}=0}^{2 n} \tilde{l}=\frac{2 n(2 n-1)}{2} \approx \frac{(2 n)^{2}}{2}=2\left(\frac{b-a}{\pi} k\right)^{2}$ і остаточно підставляючи результат підсумовування у (13), отримуємо суму по $n, n^{\prime}$ для оптичної провідності:

$\sigma=\frac{8 e^{2} \hbar^{4}}{3 \pi V_{s} m_{e}^{4} \omega^{3}}\left(1 \pm \frac{3}{5}\left(\frac{\Delta l}{b-a}\right)^{2}\right) \times$

$\times \sum_{n, n^{\prime}}\left(1-(-1)^{n+n^{\prime}}\right) k^{4}\left(k^{\prime}\right)^{2} f(E) \times$

$\times\left(1-f\left(E^{\prime}\right)\right) \delta\left(E^{\prime}-E-\hbar \omega\right)$.

На загальний об'єм оболонки зміщення ядра не впливає, тому об'єм $V_{s}$ залишається без зміни.

\section{4. Обчислення оптичної провідності}

Обчислимо отриману суму для оптичної провідності (16) у два етапи. Спочатку замінимо суму по електронних станах інтегралом, нехтуючи ефектами, пов'язаними з квантуванням електронного спектра енергій. Обчислюючи отриманий інтеграл аналогічно до [22], запишемо оптичну провідність $\sigma_{0}$ в такому наближенні у вигляді

$\sigma_{0}=\frac{32 e^{2}(b-a)^{2}}{3 \pi^{3} \hbar^{4} \omega^{3} V_{s}}\left(1 \pm \frac{3}{5}\left(\frac{\Delta l}{b-a}\right)^{2}\right) E_{\mathrm{F}}^{3} g_{\mathrm{sph}}(\nu)$,

де $E_{f}$ - енергія Фермі, величина $\nu=\frac{\hbar \omega}{E_{\mathrm{F}}}$, а функція

$g_{\mathrm{sph}}(\nu)=\int_{1-\nu}^{1} q^{3 / 2} \sqrt{q+\nu} d q=\left(\frac{(q(q+\nu))^{3 / 2}}{3}-\right.$

$\left.-\frac{\nu(2 q+\nu) \sqrt{q(q+\nu)}}{8}+\frac{\nu^{3}}{8} \ln (\sqrt{q}+\sqrt{q+\nu})\right)\left.\right|_{1-\nu} ^{1}$.

Отриманий вираз не враховує квантові ефекти, пов'язані з дискретністю електронного спектра (інформація про квантування губиться при переході від суми до інтеграла), але враховує принцип Паулі.

Тепер уточнимо вираз (17), врахувавши дискретність спектра хвильових чисел. Для цього замість заміни суми по $n$ та $n^{\prime}$, що входить у (16), інтегралом використаємо точну формулу підсумовування формулу Пуассона:

$\sum_{n=1}^{\infty} y_{1}(n)=\int_{0}^{\infty} d n\left(y_{1}(n)+2 \sum_{s=1}^{\infty} y_{1}(n) \cos (2 \pi s n)\right)$, 
де $y_{1}$ - довільна функція натурального аргументу $n$.

Для обчислення суми, що входить у (16), формулу Пуассона необхідно застосовувати двічі - при підсумовуванні по $n$ та по $n^{\prime}$. Коли взяли суму по $n^{\prime}$ потрібно застосувати формулу Пуассона до виразу, що містить дельта-функцію від дискретних індексів, а саме функції

$G(E(n)+\hbar \omega) \equiv \sum_{n^{\prime}=1}^{\infty}\left(1-(-1)^{n-n^{\prime}}\right) \times$

$\times \delta\left(E\left(n^{\prime}\right)-E(n)-\hbar \omega\right)$.

Для коректного проведення такої операції необхідно зауважити, що під дельта-функцією ми маємо на увазі границю сімейства класичних функцій (наприклад, 3 класу $C^{\infty}$ або наведеної нижче). При граничному переході площа під класичною функцією залишається рівною одиниці при прямуванні характерної ширини фігури під кривою до нуля, а її висоти до безмежності. Для наших цілей зручно застосувати сімейство класичних функції вигляду

$\delta^{*}(x)=\left\{\begin{array}{cl}0, & x<-\frac{\Delta E}{2} \\ \frac{1}{\Delta E}, & -\frac{\Delta E}{2}<x<\frac{\Delta E}{2} \\ 0, & x>\frac{\Delta E}{2}\end{array}\right.$

при $\Delta E \rightarrow 0$. Формула Пуассона застосовується до виразу, що містить $\delta^{*}$, з подальшим переходом $\Delta E \rightarrow$ 0 . Крім того, множник $\left(1-(-1)^{n^{\prime}-n}\right)$, що входить у (20), можна замінити середнім значенням - одиницею - не тільки при заміні суми інтегралом (в цьому випадку закономірність такої заміни очевидна), а й при використанні формули підсумовування Пуассона. Коректність такої апроксимації можна довести строго, розглядаючи для (20) окремо ситуації парних на непарних $n^{\prime}$. При застосуванні формули Пуассона для обох випадків отримані вирази є ідентичними.

Зробивши ці зауваження, замінимо в (20) дискретну функцію $\left(1-(-1)^{n-n^{\prime}}\right)$ на $\dddot{1} і$ середнє значення, яке дорівнює одиниці, а дельта-функцію на $\delta^{*}$ і застосуємо до отриманої суми формулу Пуассона аналогічно до того, як це було зроблено у роботі [22]. Після застосування формули Пуассона два рази (для підсумовування по $n$ та по $\left.n^{\prime}\right)$ з урахуванням $E \approx E_{\mathrm{F}}$, $E_{\mathrm{F}} \gg \theta$, де $\theta$ - температура у енергетичних одиницях, шукана оптична провідність записується

$\sigma(\omega)=\sigma_{0}(\omega)+\Delta \sigma=\sigma_{0}(\omega) \times$

$$
\begin{aligned}
& \times\left(1+\frac{2 \pi \theta\left(1-e^{-\frac{\hbar \omega}{\theta}}\right)^{-1}\left(\Phi\left(E_{\mathrm{F}}\right)-\Phi\left(E_{\mathrm{F}}-\hbar \omega\right)\right)}{E_{\mathrm{F}}^{3} \tilde{g}_{\mathrm{sph}}(\nu)}\right)= \\
& =\frac{8 e^{2}}{\pi^{4} \hbar} \frac{(b-a)^{2}}{b^{3}-a^{3}}\left(1 \pm \frac{3}{5}\left(\frac{\Delta l}{b-a}\right)^{2}\right) g_{\mathrm{sph}}(\nu) \times \\
& \times\left(1+\frac{2 \pi \theta\left(1-e^{-\frac{\hbar \omega}{\theta}}\right)^{-1}\left(\Phi\left(E_{\mathrm{F}}\right)-\Phi\left(E_{\mathrm{F}}-\hbar \omega\right)\right)}{E_{\mathrm{F}}^{3} \nu^{3} g_{\mathrm{sph}}(\nu)}\right),
\end{aligned}
$$

де функція

$$
\begin{aligned}
& \Phi\left(E_{\mathrm{F}}\right)=E_{\mathrm{F}}^{3 / 2}\left(E_{\mathrm{F}}+\hbar \omega\right)^{1 / 2} \sum_{s=1}^{\infty} \frac{\sin \varphi_{s}\left(E_{\mathrm{F}}\right)}{\operatorname{sh}\left(\pi \theta \varphi_{s}^{\prime}\left(E_{\mathrm{F}}\right)\right)}, \\
& \varphi_{s}(E) \equiv s \frac{2(b-a)}{\hbar} \sqrt{2 m_{e}}(\sqrt{E+\hbar \omega}-\sqrt{E}),
\end{aligned}
$$

поправка $\Delta \sigma$ до провідності, що враховує квантування спектра

$$
\Delta \sigma=\sigma_{0} \frac{2 \pi \theta\left(1-e^{-\frac{\hbar \omega}{\theta}}\right)^{-1}\left(\Phi\left(E_{\mathrm{F}}\right)-\Phi\left(E_{\mathrm{F}}-\hbar \omega\right)\right)}{E_{\mathrm{F}}^{3} \tilde{g}_{\mathrm{sph}}(\nu)}=
$$

$=\frac{8 e^{2}}{\pi^{4} \hbar} \frac{(b-a)^{2}}{b^{3}-a^{3}} g_{\mathrm{sph}}(\nu)\left(1 \pm \frac{3}{5}\left(\frac{\Delta l}{b-a}\right)^{2}\right) \times$

$$
\times \frac{2 \pi \theta\left(1-e^{-\frac{\hbar \omega}{\theta}}\right)^{-1}\left(\Phi\left(E_{\mathrm{F}}\right)-\Phi\left(E_{\mathrm{F}}-\hbar \omega\right)\right)}{E_{\mathrm{F}}^{3} \tilde{g}_{\mathrm{sph}}(\nu)} .
$$

Отриманий вираз для провідності (22) складається 3 двох компонент: напівкласичної провідності (17), яка не враховує дискретність спектра хвильових чисел електрона у оболонці, та малого доданка (25), що враховує названі вище ефекти. Як можна бачити, початкова сума для оптичної провідності (16) відрізняється від відповідної суми для симетричної сферичної нанооболонки [22] множником $1 \pm \frac{3}{5}\left(\frac{\Delta l}{b-a}\right)^{2}$ перед сумою. Цей множник не залежить від $n$, так що оптична провідність наночастинки відрізняється від провідності відповідної сферичної нанооболонки на такий же множник $1 \pm \frac{3}{5}\left(\frac{\Delta l}{b-a}\right)^{2}$. 


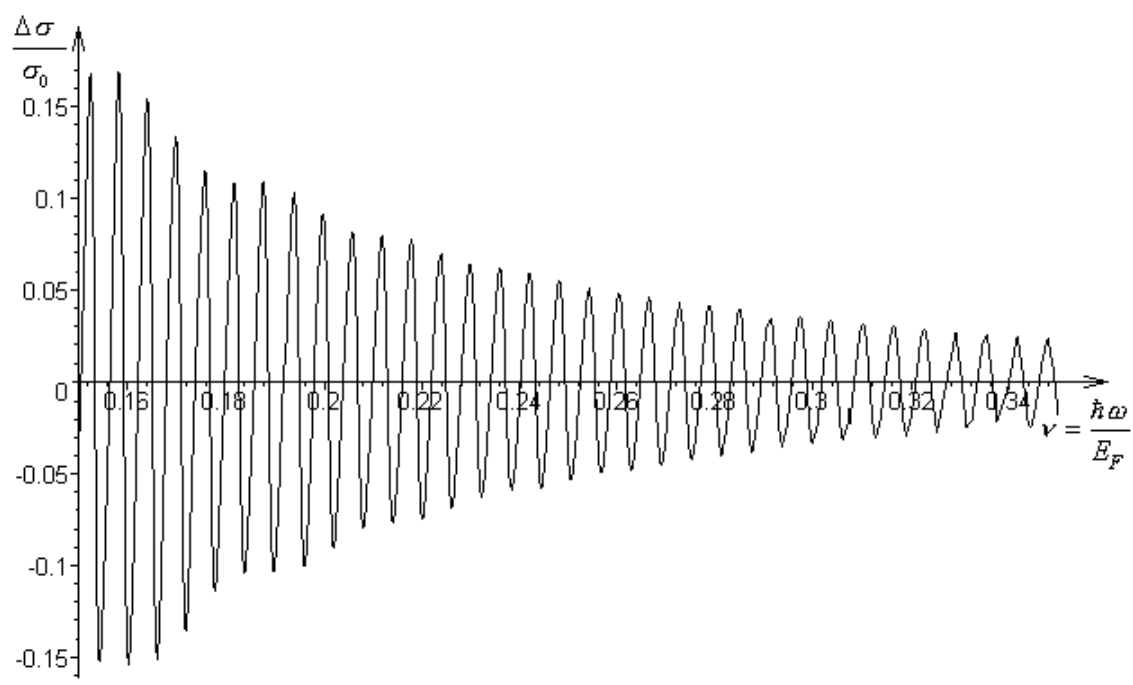

Залежність відносної квантової поправки $\frac{\Delta \sigma}{\sigma_{0}}$ від частоти світла для наночастинки 3 параметрами $E_{\mathrm{F}}=5,53$ еВ (енергія Фермі золота), радіус діелектричного ядра $a=40$ нм, середня товщина оболонки $b-a=100$ нм

Графічне представлення відносної квантової поправки $\frac{\Delta \sigma}{\sigma_{0}}$ як функції параметра $\nu$ для сферичного деформованого еліпсоїда з параметрами $E_{\mathrm{F}}=5,53 \mathrm{eB}$ (енергія Фермі золота), радіус діелектричного ядра $a=40$ нм, середню товщину оболонки $b-a=100$ нм наведено на рисунку (як можна бачити з (22), ця відносна поправка не залежить від величини $\Delta l$ зміщення центрів). 3 графіка (i з чисельних розрахунків) можна бачити, що цими квантовими поправками не можна нехтувати. Для тонших оболонок внесок цієї поправки є ще більш суттєвим. Таким чином, врахування квантування енергії електрона приводить до появи осцилюючої залежності компонент оптичної провідності від частоти світла, що падає на оболонку. Аналіз показує, що частота осциляцій залежить від товщини оболонки. Як ми вже згадували у вступі, залежність такого вигляду спостерігали навіть для відносно товстих нанодротів [14].

Таким чином, мале зміщення центрів оболонки та ядра на величину $\Delta l$ уздовж напрямку поширення хвилі приводить до появи відносного доданка другого порядку малості $\frac{3}{5}\left(\frac{\Delta l}{b-a}\right)^{2}$ у виразі для відповідної компоненти провідності. Бачимо, що ефекти, пов'язані з дискретністю спектра електрона у оболонці, та ефекти, пов'язані зі зміщенням центрів у наночастинці, впливають на оптичну провідність незалежно, так що зміщення центрів у наночастинці з дуже тонкою оболонкою (так що необхідно врахування квантування електронного спектра хвильових чисел) враховується тим же множником $1 \pm \frac{3}{5}\left(\frac{\Delta l}{b-a}\right)^{2}$ у провідності, що і для загального виразу для провідності через суму по електронних станах (16), і для напівкласичного виразу (17).

\section{5. Висновок}

У даній роботі досліджено оптичні властивості металевої наночастинки з формою, близькою до сферичної, за малого зміщення центра діелектричного ядра відносно центра зовнішньої границі оболонки. Конкретно ми отримали вираз для одноелектронної оптичної провідності такої наночастинки. Оболонка у наночастинці при цьому вважалась тонкою порівняно з розмірами наночастинки, що виконується для типових нанояєць. У результуючому виразі (22) враховані ефекти, пов'язані з дискретністю електронного спектра енергій (проявляються в осцилюючій залежності оптичної провідності від частоти). 3 результуючого виразу (22) можна бачити, що:

- зміщення центрів діелектричного ядра та зовнішньої границі наночастинки на малу величину $\Delta l$ приводить до зміни провідності наночастинки на малу відносну величину $\frac{3}{5}\left(\frac{\Delta l}{b-a}\right)^{2}$ порівняно з провідністю відповідної симетричної сферичної нанооболонки, причому оптична провідність збільшується, якщо центр зовнішньої границі оболонки зміщено відносно центра ядра у напрямку поширення хвилі, і зменшується, якщо зміщення відбувається у протилежному напрямку;

- врахування квантових ефектів, пов'язаних з дискретністю спектра хвильових чисел (i, відповідно, 
енергій) електрона, приводить до появи осцилюючої залежності оптичної провідності наночастинки від частоти світла, причому частота та відносна амплітуда осциляцій збільшуються при зменшенні товщини металевої оболонки; осцилююча залежність аналогічна до отриманої у [22];

- ефекти, пов'язані з дискретністю спектра, та ефекти, пов'язані зі зміщенням центрів, в першому порядку малості впливають на оптичні властивості наночастинки незалежно.

Як ми бачимо, відносна зміна провідності $\frac{3}{5}\left(\frac{\Delta l}{b-a}\right)^{2}$, пов'язана зі зміщенням центрів, пропорційна квадрату величини цього зміщення та обернено пропорційна до квадрата товщини оболонки. Оскільки ця поправка має другій ступінь малості, можна сказати, що оптичні властивості металевої нанооболонки є стійкими відносно малого відносного зміщення центрів ядра та оболонки і слабко реагують на таке зміщення.

За подальшого розвитку даної теми можливе дослідження сферичних наночастинок без припущення про малість відносного зміщення центра діелектричного ядра відносно центра зовнішньої границі оболонки

Автор виражає подяку доктору фізико-математичних наук, член-кореспонденту НАН України П.М. Томчуку та доктору фізико-математичних наук, член-кореспонденту АПН України Ю.І. Горобцю за увагу до роботи та корисні обговорення.

1. R.D. Averitt, S.I. Westcott, and N.J. Halas, Phys. Rev. B 58, 203 (1998).

2. R.D. Averitt, S.I. Westcott, and N.J. Halas, J. Opt. Soc. Am. 16, 1814 (1999).

3. R.D. Averitt, S.I. Westcott, and N.J. Halas, J. Opt. Soc. Am. 16, 1824 (1999).

4. L.R. Hirsch, A.M. Gobin, A.R. Lowery et al., Ann. Biomed. Eng. 34, 15 (2006).

5. F. Le, N.Z. Lwin, N.J. Halas et al., Phys. Rev. 76, 165410 (2007).

6. P. Nordlander and E. Prodan, Proc. SPIE 4810, 91 (2002).

7. E. Prodan, P. Nordlander, and N.J. Halas, Chem. Phys. Lett. 94, 368 (2003).

8. R. Chang and P.T. Leung, Phys. Rev. B 73, 125438 (2006).

9. E. Prodan and P. Nordlander, Chem. Phys. Lett. 349, $153(2001)$

10. E. Prodan and P. Nordlander, Chem. Phys. Lett. 352, 140 (2002).
11. J. Zhu, Materials Sci. Eng. A 454-455, 685 (2007).

12. K.-T. Yong, Y. Sahoo, M.T. Swihart, and P.N. Prasad, Colloids Surf. A 290, 89 (2006).

13. R.D. Averitt, D. Sarkar, and N.J. Halas, Phys. Rev. Lett. 78, 4217 (1997).

14. N. Garcia and I.L. Costa-Kramer, Europhys. News 27, 89 (1996).

15. П.М. Томчук, Б.П. Томчук, ЖЭЭТФ 112, 661 (1997).

16. Э.В. Завитаев, А.А. Юшканов, Ю.И. Яламов, ЖЭТФ 124, 1112 (2003).

17. Э.В. Завитаев, А.А. Юшканов, Оптика и спектроскопия 97, 131 (2004).

18. Э.В. Завитаев, А.А. Юшканов, Письма в ЖТФ 30, 74 (2004).

19. H. Wang, W. Yampeng, B. Lassiter et al., Proc. Nat. Acad. Sci. USA 103, 10856 (2006).

20. Y. Wu and P. Nordlander, J. Chem. Phys. 125, 124708 (2006).

21. J.B. Lassiter, M.W. Knight, N.A. Mirin, and N.J. Halas, Nano Lett. 9, 4326 (2009).

22. V.V. Kulish and P.M. Tomchuk, Surf. Sci. 602, 1045 (2008).

Одержано 17.03.11

ОДНОЭЛЕКТРОННЫЕ ОПТИЧЕСКИЕ СВОЙСТВА

НАНООБОЛОЧЕК С НЕКОНЦЕНТРИЧЕСКИМ

ЯДРОМ. УЧЕТ КВАНТОВАНИЯ

ЭЛЕКТРОННОГО СПЕКТРА

\section{В.В. Кулиш}

Р е з ю м е

Работа посвящена исследованию одноэлектронных оптических свойств (вклад одноэлектронной компоненты является существенным в областях частот, далеких от плазмонного резонанса) сферической наночастицы, состоящей из диэлектрического ядра и тонкой металлической оболочки, со слабым смещением центра ядра относительно геометрического центра всей наночастицы. Предложена модель, позволяющая записать волновые функции и спектр волновых чисел для электрона в оболочке композитной наночастицы такого типа. С помощью этой модели получены матричные элементы оптических переходов и одноэлектронная оптическая проводимость наночастицы как без учета (полуклассическая проводимость), так и с учетом квантования волновых чисел электрона в оболочке. Показано, что учет названных выше эффектов квантования приводит к появлению осциллирующей зависимости оптической проводимости наночастицы от частоты света. Показано, что влияние смещения центров и квантования спектра на оптическую проводимость наночастицы в первом приближении можно учитывать независимо. 
SINGLE-ELECTRON OPTICAL PROPERTIES OF METAL NANOSHELLS WITH A NONCONCENTRIC CORE. ACCOUNT OF ELECTRON SPECTRUM QUANTIZATION

\section{V.V. Kulish}

National Technical University of Ukraine

"Kyiv Polytechnical Institute",

Physico-Technical Institute, Chair of Applied Physics

(37, Peremoga Ave., Kyiv 03056, Ukraine;

e-mail:kulish_volv@ukr.net)

$\mathrm{S} u \mathrm{~m} m$ a r y

Single-electron optical properties of a spherical nanoparticle composed of a dielectric core and a thin metallic shell and characterized by a slight shift of the core center with respect to the geometric center of a nanoparticle have been studied in the frequency range far from the plasmon resonance, where the contribution of the single-electron component is considerable. A model that allows the wave functions and the wavenumber spectrum for an electron in the shell of a composite nanoparticle of this type to be obtained is proposed. The model is used to obtain the matrix elements of optical transitions and the single-electron optical conductivity of a nanoparticle both with and without (semiclassical conductivity) quantization of the electron energy spectrum in the shell. It is shown that the aforementioned quantization effects result in the appearance of the oscillatory dependence of the optical conductivity of a nanoparticle on the light frequency. It is demonstrated that the influences of the center shift and the spectrum quantization on the optical conductivity of a nanoparticle can be considered independently in the first approximation. 